Cinémas

Revue d'études cinématographiques

Journal of Film Studies

\title{
La transfiguration du bal
}

\section{François Jost}

Volume 15, numéro 2-3, printemps 2005

Cinélekta 5

URI : https://id.erudit.org/iderudit/012322ar

Aller au sommaire du numéro

Éditeur(s)

Cinémas

ISSN

1181-6945 (imprimé)

1705-6500 (numérique)

Découvrir la revue

Citer cet article

Jost, F. (2005). La transfiguration du bal. Cinémas, 15(2-3), 107-119.

\section{Résumé de l'article}

L'une des hypothèses admises implicitement dans la plupart des études sur l'adaptation est que le spectateur sait d'avance que le film est une adaptation, ou qu'il connaît le roman dont le film est adapté. Or, bien plus fréquemment, le film s'offre comme un substitut de roman à un spectateur qui ne l'a pas lu. Adoptant une approche pragmatique, l'article qui suit pose ces questions à propos de deux adaptations de Madame Bovary : à quoi reconnaît-on qu'un récit filmique est non seulement une transposition audiovisuelle des signes écrits, mais aussi une lecture de ceux-ci ? Y a-t-il des signes ostensibles dans lesquels se repéreraient une intention communicative, une vision de l'oeuvre? d'utilisation que vous pouvez consulter en ligne.

https://apropos.erudit.org/fr/usagers/politique-dutilisation/ 


\section{La transfiguration du bal}

\section{François Jost}

\section{RÉSUMÉ}

L'une des hypothèses admises implicitement dans la plupart des études sur l'adaptation est que le spectateur sait d'avance que le film est une adaptation, ou qu'il connaît le roman dont le film est adapté. Or, bien plus fréquemment, le film s'offre comme un substitut de roman à un spectateur qui ne l'a pas lu. Adoptant une approche pragmatique, l'article qui suit pose ces questions à propos de deux adaptations de Madame Bovary: à quoi reconnaitt-on qu'un récit filmique est non seulement une transposition audiovisuelle des signes écrits, mais aussi une lecture de ceux-ci? Y a-t-il des signes ostensibles dans lesquels se repéreraient une intention communicative, une vision de l'œuvre?

\section{ABSTRACT}

Most studies on adaptation assume that the spectator knows in advance that the film is an adaptation, or even that he or she knows the novel from which the film is adapted. Actually, more commonly the film presents itself as a substitute for the novel to a viewer who hasn't read it. In adopting a pragmatic approach, the following article asks these questions in relation to two adaptations of Madame Bovary: to what extent do we acknowledge a film script as being not only an audiovisual transposition of textual signs, but a reading of these signs as well? Are there signs that imply a communicative intentionality, a perspective of the work of art? 
S'il fallait critiquer la notion d'adaptation, je lui reprocherais volontiers d'entretenir l'illusion que les spectateurs sont d'abord des lecteurs, et qu'ils regardent un film le roman sur les genoux, arrêtant le cours de celui-là pour se plonger dans celuici, vérifiant la conformité de l'un à l'autre ou, plutôt, sa fidélité. Aussi est-il habituel de partir du littéraire pour aller au filmique.

Bien que cette façon d'aborder l'œuvre soit courante, le film s'offre bien plus fréquemment comme un substitut du roman à un spectateur qui ne l'a pas lu et qui, sans doute, ne le lira jamais. Quelques questions, qu'on néglige la plupart du temps, doivent donc être posées en priorité. Qu'est-ce qu'une adaptation pour celui qui ne connaît pas le texte qui a été transposé? Qu'est-ce que Madame Bovary pour celui qui n'a pas lu Flaubert ou qui découvre l'œuvre littéraire par l'intermédiaire du film? Et, à l'inverse, qu'est-ce qu'une adaptation pour celui qui sait qu'il est face à une adaptation? Plutôt que d'envisager deux interprétations, l'une profane, l'autre savante ou cinéphilique, on préfere généralement mesurer le savoir du spectateur au sien propre. Pourtant, de telles questions en amènent inévitablement d'autres, fondamentales d'un point de vue théorique, comme celle-ci: à quoi reconnaît-on qu'un récit filmique est, non seulement une transposition audiovisuelle des signes écrits, mais aussi une lecture de ceux-ci? Y a-t-il des signes ostensibles dans lesquels se repéreraient une intention communicative, une vision de l'œuvre?

Parmi les nombreuses transpositions qu'a connues le roman de Flaubert, deux s'imposent à cause des questions qu'elles soulèvent à propos de ces différences entre interprétation non informée, interprétation savante et interprétation cinéphilique: celles de Minnelli et de Chabrol.

\section{Celui qui ne connaît pas Flaubert...}

Partons du cas du spectateur qui va voir Madame Bovary sans avoir lu l'œuvre littéraire (celui de l'élève qui, à l'interrogation "avez-vous lu Madame Bovary?" répond: "Oui, j'ai vu le film») ou qui ignore que ce film est tiré d'un roman. De ce point de vue, quelle idée de l'auteur et de l'œuvre construisent les films de Minelli et de Chabrol? 
On remarquera d'abord que l'un et l'autre comportent une voix over (de même que Val Abraham, de Manoel de Oliveira, qui entretient des rapports lointains avec Bovary), phénomène moins courant que l'on croit et, sans aucun doute, fortement lié à l'aspect littéraire du texte d'origine. Cette voix over n'a pas le même statut chez Minnelli et chez Chabrol: elle est anonyme dans le film français, tandis que, dans le film américain, elle s'ancre dans un personnage vu au début et à la fin du film, qui n'est autre que Flaubert à son procès. D'un côté, donc, ce narrateur - extra- et hétérodiégétique —, dont l'une des caractéristiques premières est l'anonymat et l'impersonnalité; de l'autre, l'auteur en tant que personne ou personnage. Le premier n'est que le responsable des énoncés fictifs, ce locuteur L dont parle Ducrot; le second représente un être du monde, un locuteur $\lambda^{1}$. À première vue, le Flaubert de Minnelli reprend les termes du plaidoyer de Sénard, lors du procès fait au roman, et c'est le statut à la fois de l'auteur et de l'œuvre qu'il défend: «Emma est tirée de la vie, il y a des centaines de Bovary. D'autres qui ne le sont pas par manque de détermination..." Mais, outre que les questions de style évoquées au procès sont ici évacuées, on s'aperçoit bien vite que cette voix a une fonction narrative précise dans le film : faire l'économie des flashes-back, maintenir autant que possible la continuité temporelle tout en expliquant les motivations qui régissent les actes de ce que les Anglais appellent si opportunément des characters. Ainsi sont dévoilées aux spectateurs, dès le départ, ces deux pierres angulaires du récit hollywoodien: l'interprétation non ambiguë du matériau filmique et le personnage. Dès les premières scènes, tout est clair: Emma, "fleur sur le fumier", vit dans un monde d'aventures et d'amour, comme tout spectateur des films hollywoodiens, et conjugue happyness, fashion et old romance dans le rude milieu de la vie paysanne. Arrive Charles Bovary sur fond de rêve: "Elle ne sait pas que ce n'est pas un prince charmant, mais seulement un homme." Elle prend Yonville pour le paradis ("It's like a picture in a history book») et découvre, dès son mariage, la brutalité, la vulgarité, l'ivrognerie, la cruauté. Son comportement de rêveuse s'explique par la rudesse de son mari, qui dit de lui-même — pour fuir 
l'invitation à la Vaubyessard — qu'il n'est qu'un paysan (notons, en passant, que ce détail ne figure pas dans le roman).

Si l'on peut entendre dans les propos de cette voix over un écho lointain du procès, surtout si l'on en ignore la lettre, on ne manque pas de remarquer assez vite qu'elle joue un autre rôle à l'intérieur du récit de Minnelli. Loin de se contenter de justifier idéologiquement le roman, de l'extérieur, elle articule la logique immanente de la narration: elle occupe la fonction du narrateur. Ainsi, est accréditée l'idée que l'auteur et ce dernier ne font qu'un, idée que suggérait le visage de Flaubert témoignant à la cour par un récit halluciné. De trois quarts, il semble bien voir ce qu'il raconte. Dans ce récit halluciné, on chercherait pourtant vainement les "visions» de Flaubert. Le film semble montrer des événements tirés de la vie, et c'est donc l'activité romanesque elle-même qui est niée à travers cette prétention à la vérité placée en conclusion du film. Tout se passe comme si le narrateur-Flaubert ne faisait que transcrire ce qui s'est déroulé devant cette fenêtre qu'est l'écran hollywoodien (cela dit, le plaidoyer va dans ce sens).

En ce qui concerne Chabrol, il en va autrement. Le texte de la voix over qui affleure à la surface de l'image est littéraire et l'on comprend bien vite - même si l'on n'a jamais ouvert le livre - que c'est celui du roman lui-même, ou, tout au moins, celui d'un roman, en raison des effets que produit sa transposition vocale: la prosodie, le jeu des comédiens lui donnent la dignité d'un texte lu. C'est la lettre du roman qui légitime le film, et non son statut littéraire. Attentif aux occurrences des mots par rapport aux images, je remarque sans difficulté que le mode privilégié d'articulation entre les deux matériaux est l'illustration, et que le mot ne surgit que dans les cas où il est difficile d'en trouver une traduction visuelle convenable, par exemple, quand il s'agit de communiquer rapidement, et à coup sûr, un état mental («elle se rappela toutes les privations de son âme»), un sentiment («ils recommencèrent à s'aimer»), une impression («jamais Bovary ne fut si belle qu’à cette époque...»). Cet affleurement de la voix over sonne comme un aveu d'allégeance du cinéaste au romancier. Croyez-moi, nous dit-il, rien ne sera dans mon film que vous n'auriez pu lire dans le roman 
de Flaubert. Du coup, le spectateur peut se représenter le réalisateur comme un serviteur du roman et considérer que la valeur artistique du film est à la mesure de l'hypotexte hypotexte dont on promet non seulement l'esprit, mais la lettre... à l'image près!

À en juger par la narration de Flaubert telle que nous la voyons prendre corps sur l'écran, aucun doute n'est possible: il faut l'acquitter. Cet écrivain scandaleux n'obéit-il pas à toutes les règles de la réalisation et du scénario hollywoodiens? Rien qui ne soit prévisible dans le film de Minnelli: le mariage d'Emma et de Charles, annoncé dès la première séquence par un long champ-contrechamp ponctué par une musique langoureuse, puis l'aventure d'Emma avec Léon, déjà en germe dans ce regard vague du clerc de notaire le soir même de l'arrivée de l'héroïne à Yonville. Les règles hollywoodiennes justifient ici tous les déplacements et toutes les condensations. Les éléments importants pour comprendre la psychologie d'Emma, les événements, les personnages-clés sont livrés au spectateur dans le premier quart du film, comme c'est la règle dans un scénario bien construit : la présentation de Léon, la naissance de Berthe, le bal, la rencontre avec Rodolphe. Pour l'occasion, Léon accueille le couple Bovary à Yonville, où se déroulera le bal lors duquel Emma rencontrera Rodolphe. Tout élément trop choquant ou désagréable est soigneusement gommé, qu'il s'agisse de l'opération de Justin, à laquelle Charles renonce finalement de procéder ou, pire, de la mort de Charles, qui est passée sous silence. Flaubert, un parfait scénariste d'Hollywood!

En revanche, il est beaucoup plus difficile de se faire une idée de Flaubert à travers le film de Chabrol. Si celui-ci ne doit pas manquer d'humour (voir le bal où le maître d'hôtel sert une coupe de champagne à Charles, en précisant sa marque, "de Castellane", et où Charles, croyant qu'il se présente à lui, répond: «Bovary»!), son fameux réalisme, bien décrit dans tous les manuels, semble friser le fantastique gore: la mort d'Emma comme la tête du mendiant rencontré à Rouen sont des éléments du film qui n'ont rien à envier au Retour de Freddy. L'image de Flaubert que le spectateur est amené à se forger est entièrement conditionnée par l'histoire qu'il raconte. 


\section{... et celui qui a lu Madame Bovary}

Qu'en est-il, à présent, pour celui qui a lu Madame Bovary? Je répondrai à cette question par le moyen d'une analyse de l'une des scènes les plus notoires du roman: le bal de la Vaubyessard.

Du point de vue de l'action, la séquence du film de Chabrol - d'une durée totale de six minutes - commence au moment où Emma arrive dans la salle de bal («Les quadrilles avaient commencé. Il arrivait du monde. On se poussait.» — p. $84^{2}$ ), mais quelques éléments de dialogue apparaissant juste auparavant dans le roman y sont instillés («les nus-pieds vont me gêner pour danser [...]. Tu vas me chiffonner»). Manifestement, le parti pris de Chabrol est de transmettre le maximum d'informations narratives par le dialogue. Dès le début de la scène, on peut supposer que cette opération de verbalisation va perdre en route ce qui n'affleure pas dans les mots: l'impression. "On versa du vin de champagne à la glace. Emma frissonna de toute sa peau en sentant ce froid dans sa bouche» devient: "Tu m'apportes du champagne?» Faute de trouver l'équivalent visible à la description de l'invisible, le cinéaste en reste au dicible. Et la sensation passe à la trappe. De la même façon, Emma se jette dans la danse sans que l'on sente véritablement que "le cœur lui battit un peu».

Confirmant cette intuition spectatorielle, le montage ordonne la plupart des événements du roman de Flaubert en les ancrant dans le regard et l'ouïe d'Emma, par des ocularisations et des auricularisations internes secondaires: les bribes de conversations évoquant le Vésuve, le Colisée ou les clairs de lune. Tout y est ou presque, à une inversion près: la scène de l'éventail qui intervient avant que les domestiques ne cassent les vitres. Comme ces lieux de rêve dont Emma a tout juste saisi les noms, l'explication de ce geste trouve son origine dans un propos à peine perceptible — «il fait vraiment trop chaud» que l'héroïne ne semble même pas remarquer. Impassible à l'écoute de cette ambiance sonore "pleine de mots qu'elle ne comprenait pas», elle l'est tout autant devant le spectacle des domestiques qui font éclater les carreaux à coups de chaises.

Il faut dire qu'ici les vitres cassées ne donnent accès à aucun souvenir, comme c'est le cas chez Flaubert: «Bovary tourna la 
tête et aperçut dans le jardin, contre les barreaux, des faces de paysans qui la regardaient. Alors, le souvenir des Berteaux lui arrive. Elle revoit la ferme [...]. Elle était là ${ }^{3}$.» Chez Chabrol, toute l'intériorité a disparu. Le visualisable est devenu visible ou audible, il a été réduit à quelques éléments pertinents, et nous demeurons à l'extérieur du personnage, ne partageant que son point de vue perceptif, oculaire et auriculaire. Du Flaubert revu par Robbe-Grillet, en somme.

Quand Emma entre dans la danse, le principe du montage reste stable, à ceci près que Charles devient le principal foyer perceptif: il regarde sa femme danser. Néanmoins, plusieurs plans montrent le spectacle en ocularisation zéro: notamment un travelling vertical qui, partant des danseurs, s'achève sur un lustre; un autre qui cadre d'abord les jupes qui tournent pour monter ensuite au-dessus d'Emma, et enfin un troisième qui montre l'héroïne se dirigeant vers un miroir pour reprendre son bouquet et qui suggère un moment d'étourdissement.

Pour finir, c'est de nouveau Emma qui observe le bal et, en particulier, la danseuse qui ouvre le bal. L'ambiguïté du style indirect libre — «elle savait danser celle-là » — est néanmoins levée par un ancrage de cette pensée dans la bouche de Charles qui occasionne, en l'occurrence, une réplique tout droit sortie des Cousins (Chabrol, 1959) : "Elle ne doit pas rater une occasion de s'entraîner. "Si le film de Chabrol, comme le laissait prévoir l'utilisation du texte flaubertien en voix over, suit pas à pas le roman, il opère pourtant deux déplacements modaux majeurs: d'une part, la restriction du point de vue à celui d'Emma éclate au profit d'une mobilité beaucoup plus grande (d'Emma à Charles, puis de Charles à Emma); d'autre part, si, dans le roman, l'ocularisation se conjuguait à la focalisation, dans le film le spectateur est réduit à partager les regards d'Emma et à rester à l'extérieur de ses pensées, le narrateur ne dédaignant pas, à l'occasion, s'affirmer dans des ocularisations zéro, véritables résidus narratoriels.

\section{Qu'est-ce que lire une œuvre?}

Cette rupture du point de vue — interne, externe - est-elle purement gratuite ou peut-on y déceler une fonction? Le spectateur du seul film de Chabrol pencherait volontiers pour la 
gratuité. Celui qui a vu la Bovary de Minnelli accréditerait, en revanche, la seconde hypothèse. Cette hésitation nous amène à envisager une nouvelle question : qu'est-ce qu'une "lecture", une interprétation de l'œuvre? Comment se repère-t-elle dans un film et à quel niveau de l'hypotexte opère-t-elle?

À première vue, la scène du bal de la Bovary américaine est l'archétype même de la rhétorique hollywoodienne, même si elle est d'une durée inhabituelle. Sautant directement de l'arrivée d'Emma au château, accueillie par le comte d'Andervilliers, à la scène des quadrilles, la séquence, en une suite de condensations sommaires, emprunte à Flaubert, d'un côté, ce qui tient lieu d'establishing shot, et de l'autre, les quelques lignes qui annoncent la cérémonie de la danse.

Pas plus que chez Chabrol, le point de vue n'est restreint à celui d'Emma. Néanmoins, si l'on passe de celle-ci à Charles et inversement, ce n'est nullement, comme chez le cinéaste français, en raison d'une absence de point du vue, cette focalisation zéro dont parle Genette. On mesure, face à une telle scène, combien la focalisation règle moins le savoir du personnage par rapport au narrateur (ou l'inverse) qu'elle ne règle celui du spectateur par rapport au personnage. En définitive, c'est le dogme de l'omniscience, fondamental à Hollywood, qui justifie le découpage de cette séquence: en focalisation spectatorielle, cette suite de plans vise à donner à celui qui la regarde tous les éléments nécessaires à l'intelligibilité non seulement de ce moment du film, mais, plus globalement, de sa diégèse. Aussi, les deux phrases du roman concernant Charles subissent-elles une extension ${ }^{4}$ et font-elles l'objet d'un montage parallèle systématique (à noter que, pour l'occasion, c'est Charles qui saisit des bribes de conversations, tentant d'établir une communication dont il est exclu). L'alternance a ici plus qu'une valeur temporelle. Amplifiant des traits de caractère explicités auparavant par la voix over, elle assoit, ce faisant, le comportement futur d'Emma sur une opposition paradigmatique grâce/balourdise qui règle l'ensemble des actions ${ }^{5}$ depuis les premiers plans - où Emma et Charles titubent, elle de joie, lui à cause des vapeurs du vin.

Le cadrage comme l'enchaînement des plans, inféodés aux exigences de clarté aussi bien visuelle (les personnages princi- 
paux sont toujours centrés) que cognitive (on révèle leur état mental et leur être profond), modèlent bien cette séquence sur la rhétorique hollywoodienne.

En revenant au film de Chabrol, on reconnaît de multiples emprunts au film américain, qui suggèrent que l'hypotexte de la Bovary française est davantage le film de Minnelli que le roman de Flaubert. La robe d'une danseuse en gros plan, les mouvements de caméra qui partent des lustres pour arriver sur Emma; autant ces éléments paraissent gratuits chez Chabrol, autant ils sont, chez Minnelli, comme des indices ostensibles d'un parti pris : celui d'un metteur en scène de comédie musicale.

La jupe en gros plan, c'est un rideau qui s'ouvre sur le petit théâtre où va se jouer le drame d'Emma et de Charles; les mouvements d'un danseur sont réglés comme un ballet, dans lequel l'évolution de la caméra n'obéit pas simplement à des exigences de clarté, mais aussi à une recherche du spectaculaire, qui fonde le plaisir de la chorégraphie. Alors que Chabrol se contente de cadrer des couples qui valsent, chez Minnelli c'est la caméra elle-même qui tourne, véritable relais du tournis qui saisit Emma, faisant circuler cette impression de l'héroïne au spectateur: les danseurs traversent l'écran en tous sens, puis la caméra panote de droite à gauche et de gauche à droite, jouant sur les échelles de plans pour accentuer le mouvement du tournoiement. De ce point de vue, ces deux versions cinématographiques de Madame Bovary exemplifient deux façons d'adapter le littéraire: rendre visible l'écrit, ou le traduire par le biais de l'énonciation cinématographique (au sens précis que je lui donne: l'énonciation cinématographique renvoie au style du supposé réalisateur en tant qu'il "parle» cinéma ${ }^{6}$ ).

Il y a plus. En effet, bien que ces procédés soient en continuité avec la comédie musicale, on peut y déceler aussi un style qui va de Minnelli à Flaubert et de Flaubert à Minnelli.

De Minnelli à Flaubert, d'abord. Si l'alternance répond à l'obsession hollywoodienne de l'omniscience, elle constitue en outre une opposition de motifs. Car, s'il s'agit à première vue de faire comprendre au spectateur que tout sépare Emma et Charles, le montage les rapproche jusqu'à les unir dans un même destin. C'est d'abord la maladresse de Charles qui prend 
deux coupes de champagne (voir supra comment Chabrol fait une variation sur ce thème), alors que les autres convives les jettent par-dessus leur épaule, à la russe — geste enserré par un mouvement sur le lustre et par le plan d'un éventail faisant frissonner ses pendeloques. C'est ensuite Charles qui renverse un plateau rempli de verres en écho aux vitres qu'on casse afin de délivrer Emma - qui ne peut plus respirer - de son oppression. Et enfin, les deux époux titubant, l'un d'aise, l'autre d'ivresse, les deux séries se rejoignent pour clore la scène lorsque Charles, visiblement saoul, vient s'interposer entre Emma et son cavalier pour inviter celle-ci à danser.

Le début de la scène suivante mène à terme la rupture entamée depuis la scène du bal: Emma tient les rennes de la calèche que Charles conduisait quelques minutes plus tôt (dans la nuit) et mène l'attelage jusqu'à Yonville. Comment ne pas y voir une métaphore lourde de sens pour la suite, où Emma fera cavalier seul, si j'ose dire, au cours de cette scène où Rodolphe lui propose de faire du cheval?

De Flaubert à Minnelli, maintenant. Moins attentive aux détails narratifs que celle de Chabrol, toute la mise en scène du cinéaste américain exemplifie, en effet, une impression: l'espèce de magie qui émane de la danse pour Emma.

Dès ses premiers pas, Minnelli parvient à rendre visuellement cette incroyable allégresse qui envahit l'héroïne. Conjuguant un mouvement de caméra et un trucage - à la façon de La belle et la bête de Cocteau -, il donne au spectateur l'impression que l'héroïne glisse plus qu'elle ne danse («mais bientôt l'émotion disparut: et se balançant au rythme de l'orchestre, elle glissait en avant avec des mouvements légers du cou» - p. 84). Tous les plans sur les lustres - outre la fonction paradigmatique qu'on leur a reconnue - sont au service de cette impression concentrée dans les mots de Flaubert: "Ils tournaient, tout tournait autour d'eux, les lampes, les meubles, les lambris, et le parquet, comme un disque sur un pivot» (p. 86). En ce sens, la mise en scène est imprégnée de Flaubert.

Un autre plan, que j'ai négligé bien qu'il occupe une place centrale, confirme cette intuition: celui où Emma se voit dans un miroir, entourée d'hommes. Outre que, du point de vue 
temporel, il est placé à l'exact hémistiche de la séquence (qui débute 5 min $10 \mathrm{~s}$ avant et se termine 5 min $10 \mathrm{~s}$ après), il est le pivot autour duquel tourne la suite des événements et sur lequel s'articule la psychologie d'Emma. En voyant son image de femme courtisée, elle acceptera la danse qu'elle a d'abord refusée au vicomte. Si les images illustrant la contradiction de l'héroïne sont symétriquement disposées autour de cette révélation spéculaire, elles renvoient aussi, par un parallèle rigoureux, à cet échange de répliques à propos de Charles: "... some doctor's wife. - Oh, yeah, the peasant.» Après ce dédoublement d'images qui fait basculer la vie d'Emma, nous pouvons voir la réalité à travers ses yeux: les lustres tournent en ocularisation interne primaire, confirmant le rôle central que ce motif occupe dans la lecture de Flaubert par Minnelli. Les défenseurs de la thèse de la fidélité objecteront que le miroir - pour séduisant qu'il puisse être ne se trouve nullement dans le roman. Du moins pas à cette place. Car il suffit de poursuivre sa lecture pour constater le rôle que Flaubert lui confere: juste après la scène où Emma devient la maîtresse de Rodolphe, il écrit: «[...] mais, en s'apercevant dans la glace, elle s'étonna de son visage. Jamais elle n'avait eu les yeux si grands, si noirs, ni d'une telle profondeur. Quelque chose de subtil épandu sur sa personne la transfigurait» (p. 191). C'est bien au spectacle de cette transfiguration que le film nous convie, juste avant qu'Emma ne tombe dans les bras de Léon. Chez Flaubert, la métamorphose a lieu dès avant le bal, mais pour Charles seulement: «Il la voyait par derrière, dans la glace, entre deux flambeaux. Ses yeux noirs semblaient plus noirs» (p. 84). Quant à Emma, il lui faudra Rodolphe et plus de cent pages pour s'en apercevoir. Pour Minnelli - rhétorique hollywoodienne oblige - l'amour est dès le départ donné comme une suite de ses rêves. Malgré cette différence, les voies de ce dévoilement telles que Minnelli les trace vont conduire directement au roman. Le lecteur requis n'est plus, comme chez Chabrol, celui qui avance page à page, dans l'ignorance de ce qui va advenir (quel «événement!», comme dit Emma avant le bal), mais un lecteur relisant Madame Bovary, attentif aux rimes et échos du texte. En l'occurrence, la condensation qu'opère le cinéaste américain est une condensation que j'appellerai volontiers omnisciente. 
Les Bovary de Minelli et de Chabrol entretiennent avec le roman des relations paradoxales. À première vue, en effet, le film américain ramène la création littéraire à une transcription de la réalité, en présentant le romancier comme un simple spectateur prenant des notes sur ce qu'il voit, tandis que le film français garde ostensiblement la trace de Flaubert écrivain, en citant sa prose. Pourtant, à y regarder de plus près, l'adaptation de Chabrol se révèle beaucoup moins soucieuse de la lettre que du contenu narratif, et celle de Minelli plus préoccupée de traduire des impressions que de respecter la chronologie des événements. L'image de l'auteur que les deux films construisent est donc très différente selon le degré de connaissance préalable que le spectateur a du texte de départ et de l'histoire du cinéma. Si la Bovary de Minelli peut, aux yeux du spectateur profane, sembler passer par-dessus le texte de Flaubert, au contraire, elle apparaîtra au flaubertien comme une tentative accomplie de restitution de l'impression littéraire. À l'inverse, la Bovary de Chabrol, manifestement plus littéraire pour celui qui n'a pas lu le roman, en raison de ses longues citations, pourra paraître fort loin du style du roman au même flaubertien. Quant au cinéphile, il préférera y voir une succession de clins d'œil à Minelli... Comme on le voit, le chassé-croisé entre lecture savante et lecture profane est sans doute plus complexe qu'on pouvait le penser au premier abord.

\section{Université Paris III}

\section{NOTES}

1. Le locuteur L, selon Ducrot (1984), est celui qui est responsable de l'énonciation en tant que telle. Le locuteur $\lambda$ est le locuteur comme être du monde.

2. La pagination renvoie à Flaubert 1986.

3. Comme on le sait, cette scène constitue, dans le roman, l'élément premier d'une téléstructure jouant comme un stimulus. Voyant une tête d'homme à travers une fenêtre, le bal de la Vaubyessard s'animera dans la tête d'Emma (p. 98).

4. "Charles se traînait à la rampe, les genoux lui rentraient dans le corps. Il avait passé cinq heures de suite, tout debout devant les tables, à regarder jouer au whist sans y rien comprendre."

5. Au passage, on observera que se trouvent ici mêlées les valeurs de ce que Metz appelait le syntagme alterné et le syntagme parallèle. Le critère qui chez lui les oppose - chronologie vs a-chronologie - est donc loin d'être une frontière infranchissable.

6. Cf. Jost 1992. 


\section{RÉFÉRENCES BIBLIOGRAPHIQUES}

Ducrot 1984: Oswald Ducrot, Le dire et le dit, Paris, Minuit, 1984.

Flaubert 1986: Gustave Flaubert, Madame Bovary, Paris, Garnier-Flammarion, 1986. Jost 1992: François Jost, Un monde à notre image. Énonciation, cinéma, télévision, Paris, Méridiens Klincksieck, 1992. 